\title{
Cosmologists look forward to clearer picture
}

Tony Reichhardt, Washington

The first data from the Wilkinson Microwave Anisotropy Probe (WMAP) were released last week, some eight years after the mission was first proposed to NASA. The analysis of the data, by contrast, is happening much faster it is already appearing on preprint servers.

The satellite, which has now spent more than a year surveying the sky at a distance of some 1.5 million kilometres from Earth, measures variations in the temperature and polarization of the cosmic microwave background $(\mathrm{CMB})$, the faint radiation dating from the dawn of the Universe.

In an analysis done before the data were released, the WMAP team produced the best estimates yet of key cosmological parameters.

The new data imply an age for the Universe of 13.7 billion years, and a distribution of mass and energy in which $4 \%$ of the Universe is normal matter (atoms), 23\% is dark matter, and $73 \%$ is dark energy. These figures fit well with previous estimates. The age at which the first stars seem to have formed - 200 million years after the Big Bang - is significantly earlier than most astronomers had thought.

"The really huge impact is that they tightened all the screws and bolts, and hammered everything with a superb precision," says Max Tegmark, a cosmologist at the University of Pennsylvania in Philadelphia. As a result,

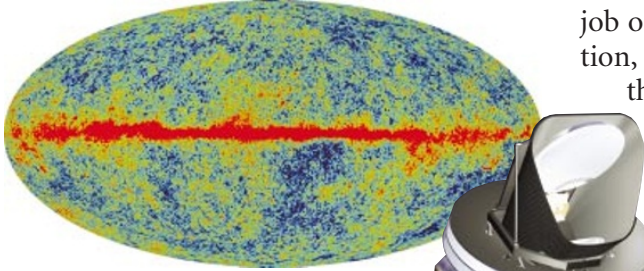

job of characterizing the foreground radiation, which can mask the faint signal from the microwave background.

Before then, several ground-based studies will examine small patches of the sky with even greater resolution. Among these are the South Pole Telescope, led by the University of Chicago, and

The WMAP probe's images of cosmic microwaves (above) are set to be bettered by the Planck satellite (right).

he says, cosmology has taken "a giant leap forward in credibility", and "has been transformed into a real hard science".

Soon after the data were released on $11 \mathrm{Feb}-$ ruary, papers began appearing on the arXiv preprint server operated by Cornell University. A group led by Carlos Martins of the University of Cambridge used the WMAP data to investigate the value of fundamental constants in the early Universe. Others compared the data to previous sky surveys and assessed its impact on theories of the geometry of the Universe. "I predict that you'll see a thousand other papers in the coming years," says Tegmark.

The next big step in all-sky CMB mapping will be the European Space Agency's Planck satellite, due to launch in 2007. Planck will have three times the resolution of WMAP, and it should do a more thorough the Chilean-based Atacama Pathfinder Experiment, proposed by the Max Planck Institute for Radioastronomy in Bonn. Their images of the microwave background may yield new information on the period when the first stars formed.

Another goal for future investigations is a more precise measurement of the polarization of the microwave background, discovered last year by the Degree Angular Scale Interferometer in Antarctica. The WMAP also measured this polarization, and a host of ground- and balloon-based instruments will take the investigation a step further.

One such balloon-based experiment, called BOOMERANG, recently completed a flight in Antarctica, where it collected "beautiful data" on the polarization with greater sensitivity than WMAP, according to principal investigator Andrew Lange of the California Institute of Technology. He expects to publish the results later this year.

http://arxiv.org

\section{Windfall spurs rare Israeli-Palestinian research effort}

\section{David Adam, London}

Most collaborations between Israeli and Palestinian scientists have fallen victim to the upsurge of violence that has disrupted the region in recent years. But one surviving partnership - an investigation of the hereditary basis of deafness among people in the region - last month received a welcome boost: a donation of US $\$ 100,000$ from a \$1-million prize scooped by geneticists John Sulston, Sydney Brenner and Robert Waterston.

The research is led by geneticists Karen Avraham, an Israeli at Tel Aviv University, and Moien Kana'an, a Palestinian who works at Bethlehem University on the West Bank Kana'an says that they have already recruited some 75 Palestinian families for the project, which is aimed at mapping the genes that cause their society to have an unusually high rate of genetic deafness. Avraham hopes that Sulston's donation will allow them to bring in new postgraduate students in October to join Hashem Shahin, a Palestinian PhD student who has worked in her lab since 1998.

The donation comes from the winnings collected by Sulston, Brenner and Waterston after being awarded one of the three Dan David Prizes in 2002. These prizes, set up by Dan David, president of photo-booth operators Photo-Me International, recognize outstanding "scientific, technological, cultural or social impact on our world". The award's winners are compelled to give $10 \%$ of their prize to younger colleagues in their field.

"We've wanted to expand the scheme for years," Avraham says. It was the political situation rather than a lack of funds that was putting the duo off, she adds, but Sulston's move has persuaded them to take the plunge. "It's a validation of what we're doing and has given us hope," she says.

Both Avraham and Kana' an stress that their cooperation is for the benefit of science. "The collaboration itself was not a political decision," Kana'an says. "It started at a scientific grassroots level and has developed from there." Kana'an is the only Palestinian researcher investigating the problem locally, although several international groups have visited the region to take DNA samples.

One feature of the study is that many of the families recruited are very large and have more than one affected member, making it easier to track common genes that may be responsible. "Very large families are incredibly useful in this type of research," says Karen Steel, who studies hereditary deafness at the Medical Research Council's Institute of Hearing Research in Nottingham, UK. “They are a very powerful resource for gene mapping."

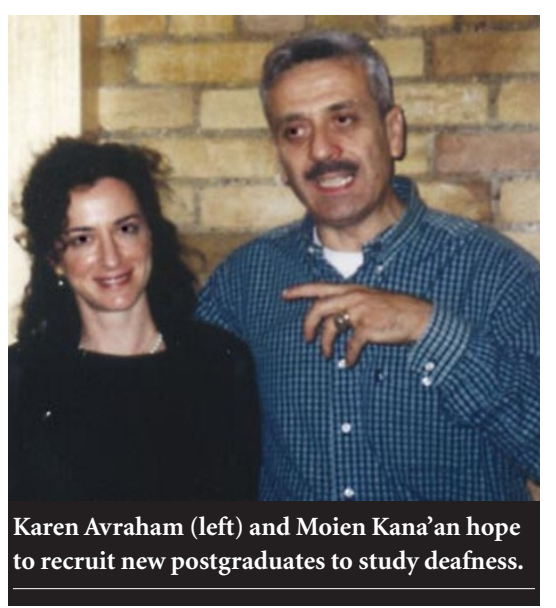

